\title{
The effect of age, gender and noise sensitivity on the liking of food in the presence of background noise
}

\author{
Mahmoud A. Alamir ${ }^{*}(\mathrm{a})$, Aws AlHares ${ }^{(\mathrm{b}, \mathrm{c})}$, Kristy L. Hansen $^{(\mathrm{a})}$, Ahmed Elamer $^{(\mathrm{d}, \mathrm{e})}$ \\ (a) College of Science and Engineering, Flinders University, Clovelly Park, Adelaide, \\ SA 5042, Australia. \\ (b) Department of Accountancy and Finance, Business School, University of \\ Huddersfield, UK. \\ (c) Faculty of School of Business Studies, College of the North Atlantic in Qatar, Qatar. \\ (d) Brunel Business School, Brunel University London, Kingston Lane, London, UK. \\ (e) Accounting Department, Faculty of Commerce, Mansoura University, Egypt. \\ mahmoud.alamir@flinders.edu.au; mahmoudelamir2011@gmail.com Tel.: +61 451052313
}

\section{Highlights}

- The effects of age, gender and noise sensitivity on the liking of food were analyzed at different types and levels of background noise and significant differences were found.

- The noise-sensitive group had lower food liking ratings than the insensitive group.

- Females had lower liking ratings of food than males.

- The younger age group of participants gave higher liking ratings of food than the older age group.

- Theories of the perceived liking of food in the presence of background noise due to the factors studied were discussed.

- The results highlight how non-acoustic factors affect food perception, providing invaluable information for food providers.

No of words: 6492 


\begin{abstract}
The liking of food in the presence of background noise has been associated with its type and level. So far, however, there has been little studies investigating the non-acoustic factors associated with food perception in the presence of background noise. This study investigated the food liking due to three non-acoustic factors (i.e. gender, noise sensitivity and age) in the presence of background noise, relative to the ambient background noise (i.e. no noise conditions). Fifteen participants rated the liking of food via questionnaires. The perceptual relative food liking and its explaining theories due to age, gender and noise sensitivity at different noise types and levels were presented. The results indicated that age, noise sensitivity and gender influence relative food liking. Females had lower liking ratings of food than males $(p=0.038)$. Noise sensitivity was also negatively correlated with the relative liking of food $(r=-0.72, p<0.001)$. Sensitive participants gave lower relative food liking ratings $(p=0.023)$. The older participants also gave lower relative food liking ratings $(p=0.01)$. The findings could enhance the models and theories of food perception due to background noise by including both the acoustic and non-acoustic factors. A better understanding of these factors effects on food perception can be an important area of interest in noise management of dining areas. They will also lead to future practical and educational applications. These include a better service that could be presented from food providers and more practical acoustic design of dining areas to suit different groups of people.
\end{abstract}

Keywords: Background noise; Confounding factors; Food perception; Psychology; Psychophysics. 


\section{Introduction}

\subsection{Background}

Noise emissions were reported to be a major cause of complaints after poor service during the dining experience (Spence, 2014; Spence et al., 2019). Moreover, there are increasing levels of noise from different noise sources such as road traffic noise in restaurants, public places and homes (Münzel et al., 2018b; Pawlaczyk-Łuszczyńska et al., 2018; Spence, 2014).

Many symptoms can be elicited due to exposure to noise. These include annoyance, sleep disturbance and stress (Mathias Basner, Wolfgang Babisch, Adrian Davis, Mark Brink, Sabine Janssen, Stephen Stansfeld, 2014). They could develop over time and may cause hypertension, obesity, diabetes and cardiovascular disease (Münzel et al., 2018a; Poulsen et al., 2018). Food enjoyment can be also affected in the presence of background noise (Spence, 2014; Spence et al., 2019).

\subsection{Theories of food perception in the presence of background noise}

Background noise could affect the behaviour of food and drink through physical, psychological and physiological pathways (Duizer, 2001; Spence, 2014; Woods et al., 2011). Sound can elicit negative or positive emotional responses depending on small differences in its physical properties, context and individual traits of the person exposed to the sound (Alamir et al., 2019; Fastl and Zwicker, 2001). These emotional responses were found to be correlated with the perception of food (Kantono et al., 2019). The levels and types of noise could also affect the perception of a gustatory cue. Crisinel and Spence (2010) showed that the pitch of sound was associated with different tastes of food. For example, low pitch sounds were associated with bitter and salty foods, while high pitch sounds were associated with sweet and sour foods. Another possible explanation 
could be that noise-induced stress makes people eat or drink more to distract themselves from that noise (Woods et al., 2011). Wesson and Wilson (2010) have speculated that sound could have a direct effect on odour transduction, which in turn could affect the perception of food.

\subsection{The effect of non-acoustic factors on food perception}

Apart from acoustic characteristics which can affect the perception of the food considerably such as level and type of noise (Kantono et al., 2016b; Spence, 2014), non-acoustic factors could potentially affect food perception in the presence of background noise. Kantono et al. (2019) reported that emotional and electrophysiological measures could correlate with the perception of gelato in the presence of different types of music. Electrophysiological measures (in terms of skin conductance, blood volume and heart rate) were correlated with the type of music and perception of food. Liked music elicited positive emotions, while disliked music-evoked negative responses. This could be explained that sounds could elicit specific parts of the brain responsible for positive emotions and reward (Kantono et al., 2019). The subjective emotional responses to noise can be also mediated by the stimulus acoustic characteristics, context and individual traits (Alamir et al., 2019). Emotions were found to be correlated with food choice and consumption (Macht, 2008).

Human psychological factors can affect food liking ratings. For example, gender can modulate food perception. Michon et al. (2010) examined the effect of gender on the liking of food without including background noise. They found that males had higher food liking ratings than females. Older people generally have a lower taste ability as compared to younger participants (Kremer et al., 2007; Mojet et al., 2001; Rolls, 1999; Ship, 1999).

However, much uncertainty still exists about the relation between some non-acoustic factors (e.g. age, gender and noise sensitivity) and the liking of food or other food perception responses in the 
presence of the masking background noise. This indicates a need to understand the various perceptions of food that exist due to these non-acoustic factors in the presence of the background noise.

\subsection{Study Aims}

This study aims to contribute to this growing area of research by exploring how three non-acoustic factors (i.e. age, gender and noise sensitivity) could affect the liking of food in the presence of the masking background noise. This could help develop models and theories of food perception in the presence of the masking background noise by including the non-acoustic factors such as age, gender and noise sensitivity besides the acoustic factors such as noise type and level. Understanding the effects of these factors on food perception can also provide us with insights into how we can manage noise in dining areas. This also provides practical insights into understanding how non-acoustic factors can affect food perception to provide adequate education for food providers.

The background noise can be classified as "masking background noise" and "ambient background noise". Masking background noise represents the noise from major noise sources (e.g. road traffic noise or music) (Fastl and Zwicker, 2001), apart from the background noise found originally in the dining area (i.e. no noise conditions from noise sources, shortly "the ambient noise" used throughout this paper ). 


\section{Methods}

A laboratory non-focused listening test was done based on a repeated measure design in which all participants rated all stimuli.

\subsection{Participants}

Fifteen participants (6 males and 9 females) rated their response to food liking. They were all English speakers and were between 22 and 45 years old (mean: 31.28, SD =7). All participants had normal hearing threshold levels. This was checked through a questionnaire (Alamir et al., 2019; Schäffer et al., 2016).

The degree of participants' sensitisation to noise is an important factor in noise perception (Gille et al., 2016a). Participants' sensitivity scores were collected to study the effects of sensitisation to noise on food liking in the presence of background noise. To obtain these scores, participants completed the 21-question noise sensitivity survey originally designed by Weinstein (Weinstein, 1978).

\subsection{Noise stimuli}

The participants rated nine masking background noise stimuli (three types at three levels) besides the ambient background noise in the listening room. The masking background sound types were relaxing music, restaurant noise and road traffic noise. We used recorded masking background noise stimuli of restaurant noise and road traffic noise rather than being synthesised.

An SVAN 979 sound level meter was used to record and calibrate the noise samples and the ambient background noise in the room. To do this, it was firstly calibrated using an acoustic calibrator from the type SV 30A at a level of $94 \mathrm{~dB}$ and a frequency of $1000 \mathrm{~Hz}$.

The noise levels of the masking background noise included in these experiments were 30, 40 and $50 \mathrm{dBA}$. The lowest masking background noise level of $30 \mathrm{dBA}$ was chosen to be noticeable above 
the ambient background noise of $22 \mathrm{dBA}$. The highest masking background noise level of $50 \mathrm{dBA}$ was also chosen to protect participants from higher levels. The measured spectra of the sound samples are shown in Fig. 1. It can be shown that all samples contain tonal components at low frequencies. However, relaxing music contains more tonal components over the frequency range. The ambient background noise in the room was $22 \mathrm{dBA}$ and only audible at high frequencies as shown in Fig. 1 when compared with the ISO hearing threshold levels (ISO 226:2003) (ISO 226:2003 - Acoustics -- Normal equal-loudness-level contours, 2003). The background noise in the listening room was used as a baseline comparison for rating the liking of food. The uncalibrated masking and ambient background noise recordings can be accessed through SoundCloud (https://soundcloud.com/mahmoud-alamir-682790423/sets/background-noise-samples).

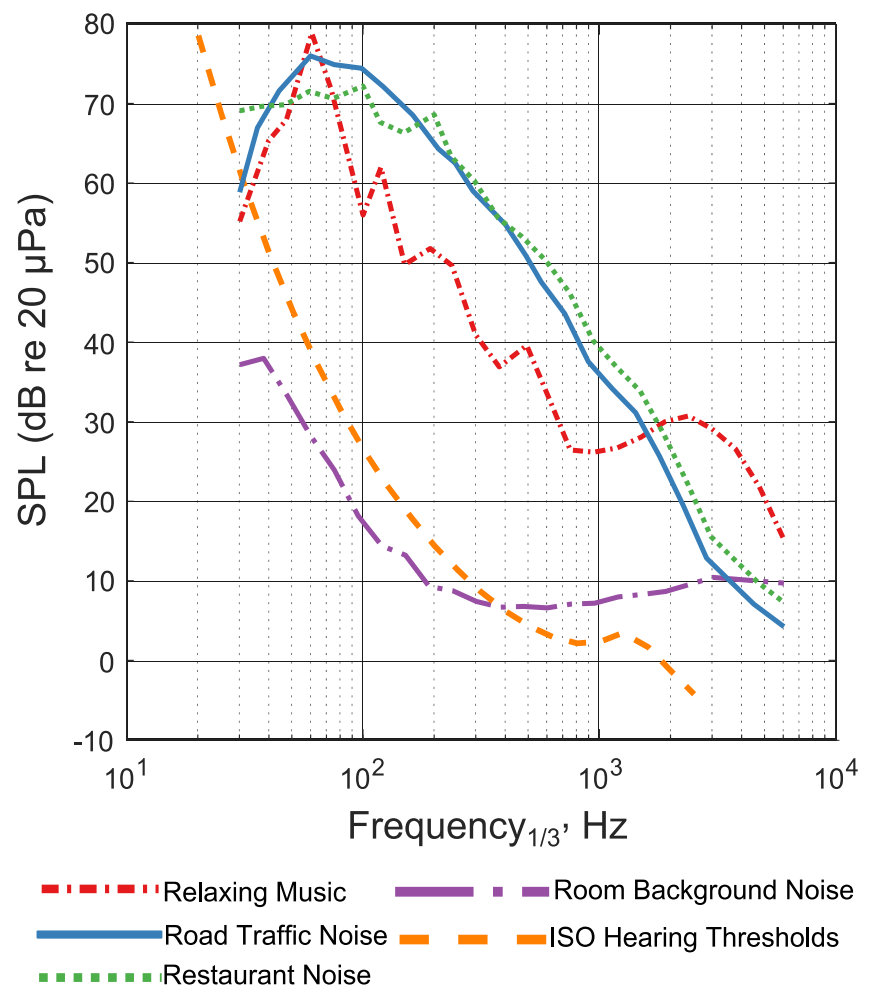

Fig. 1. Third-octave band spectra for masking background noise samples used in these experiments at $30 \mathrm{dBA}$ and ambient background noise in the listening room at $22 \mathrm{dBA}$, compared to the ISO hearing threshold levels (ISO 226:2003 - Acoustics -- Normal equal-loudness-level contours). The y-axis represents sound pressure levels (SPLs) corresponding to third-octave band frequencies on the x-axis. 


\subsection{Noise reproduction}

The masking background noise stimuli were reproduced using four loudspeakers of type (ASPIRE IC6-W-T, designed by Crestron), which were fixed on the ceiling of the room. The input to the loudspeaker was obtained from an amplifier of type (Lab Gruppen), which was connected to a computer. Sound calibration was carried out to ensure that the overall SPL was faithfully reproduced at the participant's head position.

\subsection{Food stimuli and presentation}

All participants had the same food samples served in two separate plates and presented at the same time. One plate had one falafel sandwich and the other plate contained two rainbow fruit skewers. These food samples were commercially available and provided by (https://platters.com.au/). They started eating after hearing the stimuli onset.

\subsection{Test procedures}

The listening test commenced with a training session. In that session, they were trained on how to give a rating using a laptop in the presence of a random sample of the masking background noise stimuli. The ratings of training stimuli were excluded from the analysis. After that, the participants rated nine masking background noise stimuli (three types at three levels) besides the ambient background noise in the listening room. A rest period followed each stimulus to give participants time to rate the samples, which were played randomly. This period was self-determined by the participants with a minimum duration of 15 seconds.

After each stimulus had stopped playing, participants were asked to give a rating of the liking of the food through questionnaires. The judgment of food liking was given on an 11-point Likert scale. It was a linear scale, which has discrete values from " 0 " to " 10 " with a step of " 1 ". The extreme alternatives were verbally labelled as "Not at all" and "Extremely". 
The research plan and methodology for these experiments have been accepted by the Social and Behavioural Research Ethics Committee (SBREC) at Flinders University.

\subsection{Statistical analysis}

A mixed-model analysis was used to examine fixed effects of sound pressure level, type, gender, noise sensitivity and age and their interaction using an autoregressive covariance structure (AR1) to adjust for serial correlation across trials (Littell et al., 2000). SPSS software was used in this analysis (IBM SPSS Statistics for Windows V. 25, IBM Corp, Armonk, NY). The fully saturated model was run first, followed by removal of non-significant 3-way and 4-way interaction terms. Significant interaction and main effects were examined in more details using Bonferroni adjusted pairwise contrasts within the mixed model.

The correlations between age and noise sensitivity and the response were calculated using the Pearson product-moment correlation. Pearson product-moment correlation was also used to find the correlation between each gender and the liking of food at the three levels studied.

A statistical power analysis was performed for sample size estimation, based on a pilot study with 6 participants, comparing the effects of noise type, its level, age, gender and noise sensitivity on the liking of food in the presence of background noise. The effect size was considered to be medium using Cohen's d criteria. With an alpha $=0.05$ and power $=0.80$, the projected sample size needed with this effect size was approximately $\mathrm{N}=14$ for this simplest within-group comparison. Thus, our proposed sample size of 15 was proposed to be adequate for the main objective of this study.

A normal distribution split was used to identify analytical differences between different groups of participants based on their sensitivity score and age (DeCoster et al., 2009; Iacobucci et al., 2015). Participants with a sensitivity score higher than the mean sensitivity score were classified as 
sensitive. These classification procedures were also used to classify the participants into two age groups.

The relative ratings of food liking were reported in this paper. To obtain these, the rating of the ambient background noise (i.e. no masking noise condition) was subtracted from the food liking ratings of the masking background noise samples for each participant. 


\section{Results}

\subsection{The effect of gender on the relative food liking ratings}

The gender had a significant effect on the relative food liking ratings $(p<0.001)$. Females had lower food liking ratings than males (mean [95\% CI]: $0.78[0.05 ; 1.52], p=0.038$ ) as shown in Fig. 2. A negative correlation between sound level and perceived liking of food was obtained for both genders $(r$ (females $)=-0.46, p<0.001, r$ (males $)=-0.54, p<0.001)$ as shown in Fig. 3.

(a) Males

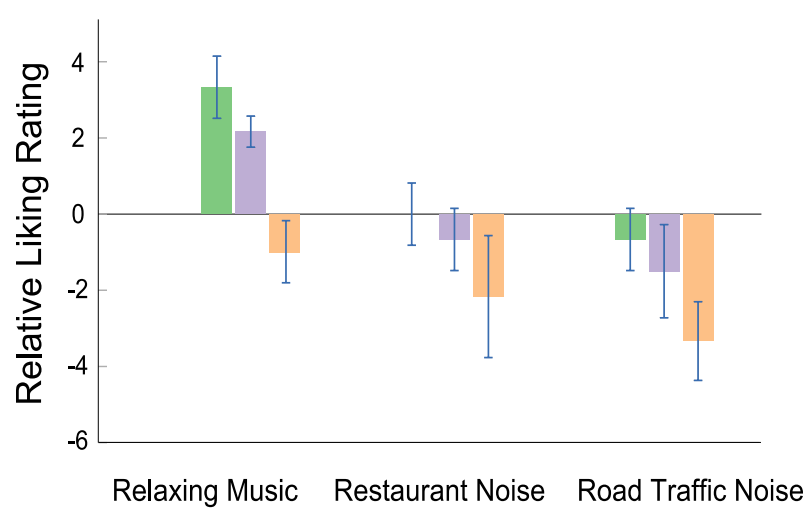

(b) Females

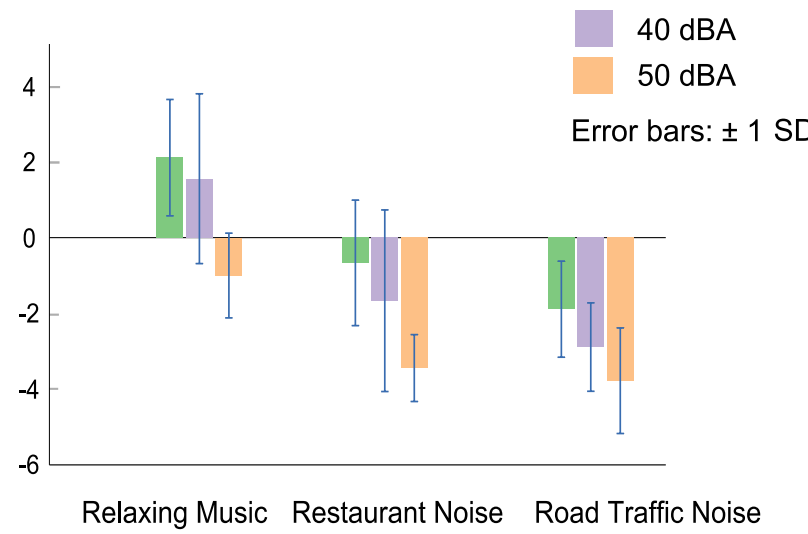

Fig. 2. Food liking ratings of three masking background noise types at three sound pressure levels, relative to the background noise in the listening room for (a) males (b) females.

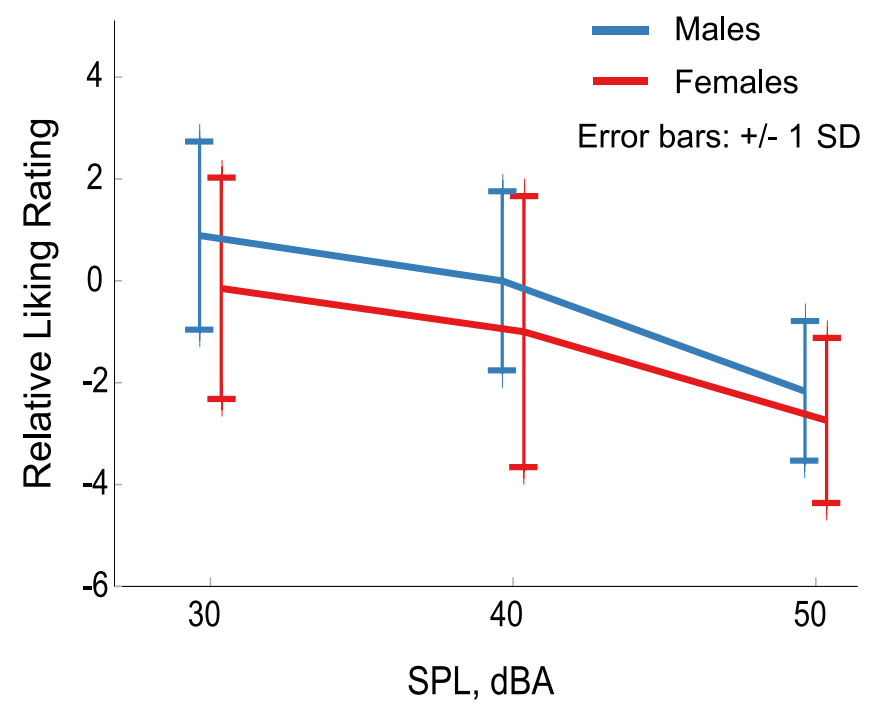

Fig. 3. A comparison between males and females of food liking, relative to the ambient background noise in the listening room at three sound pressure levels $(\mathrm{SPLs})(r$ females $)=-0.46, p<0.001, r($ males $)=-0.54, p<0.001)$.

\subsection{The effect of noise sensitivity on the relative food liking ratings}


The effect of noise sensitivity on the perception of food liking was significant $(p<0.001)$. A negative correlation between noise sensitivity and the perceived relative ratings of the liking of food was obtained $(r=-0.72, p<0.001)$ as shown in Fig. 4 .

Participants were classified into sensitive and insensitive groups based on the procedure shown in Fig. 5a. Participants with a sensitivity score higher than $46.67 \%$ were classified as sensitive. Fig. $5 \mathrm{~b}$ shows the differences between the sensitive and insensitive groups. The sensitive group had lower relative food liking ratings than the insensitive group, and the two groups had significantly different ratings of the food liking $(0.86[0.12 ; 1.59], p=0.023)$.

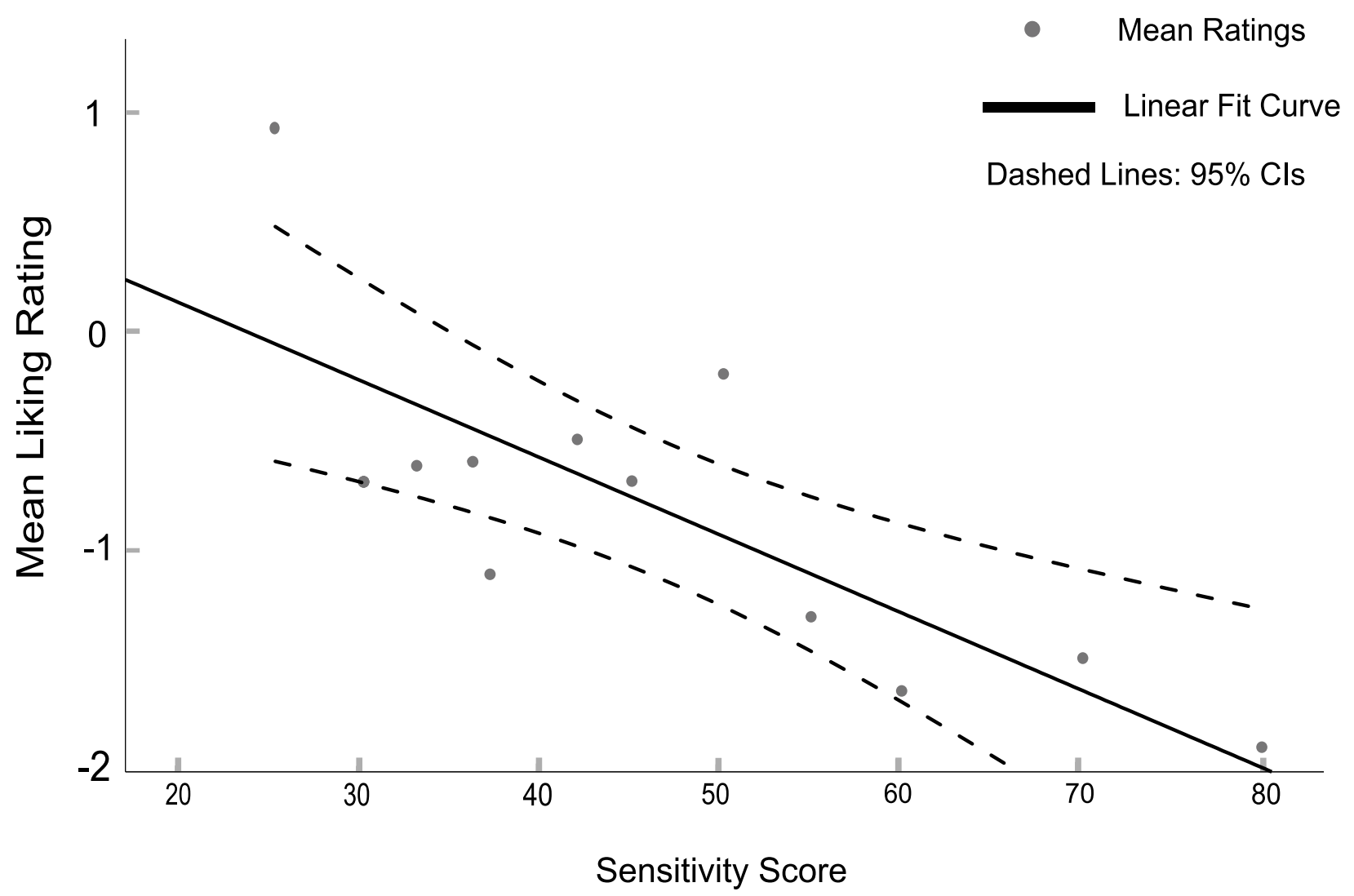

Fig. 4. The relationship between noise sensitivity score and the liking of food, relative to the background noise in the listening room $(r=-0.72, p<0.001)$. Points represent the mean of all ratings at the sensitivity score on the $\mathrm{x}$-axis. 
(a)

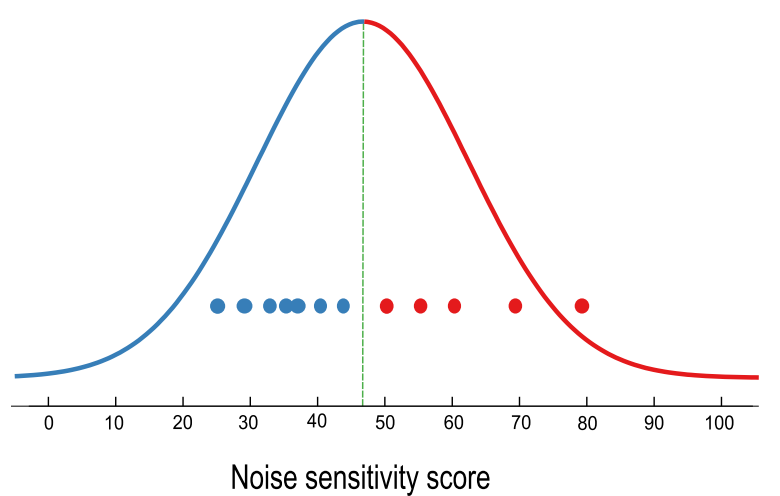

(b)

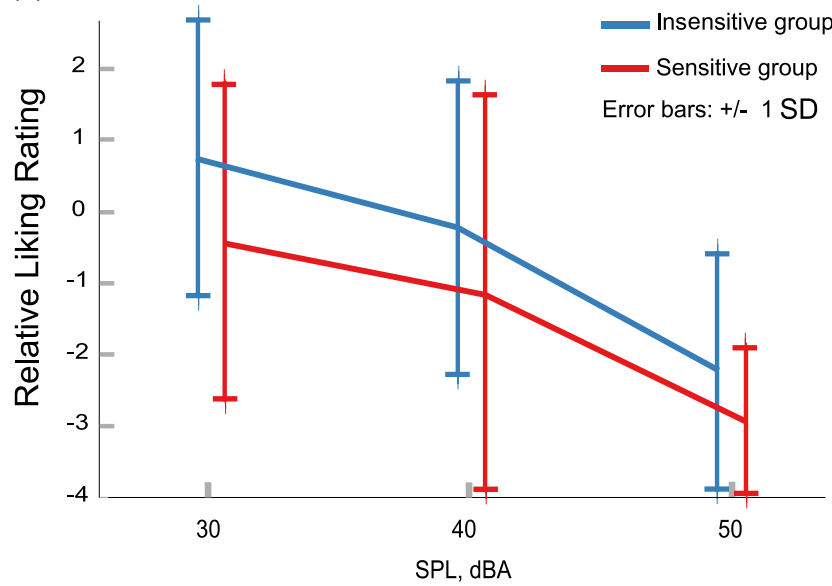

Fig. 5. (a) Noise sensitivity scores and associated normal distribution showing how participants were classified as either sensitive or insensitive. (b) Relationship between relative liking of food and sound pressure level (SPL) for sensitive and insensitive participants.

\subsection{The effect of age on the relative food liking ratings}

Age has a significant effect on the perception of noise $(p<0.001)$. Fig. 6 shows that an increase in age led to a decrease in the liking of food $(r=-0.63, p<0.001)$.

Participants were also classified into two groups based on the procedure shown in fig7a. Participants older than around 32 years old were classified as group 2, while participants younger than 32 years old were classified as group 1. Fig. $7 \mathrm{~b}$ shows the differences between the two groups.

The older group (group 2) had lower food liking ratings than the younger group (group 1) (0.97 $[0.23 ; 1.7], p=0.01)$. 


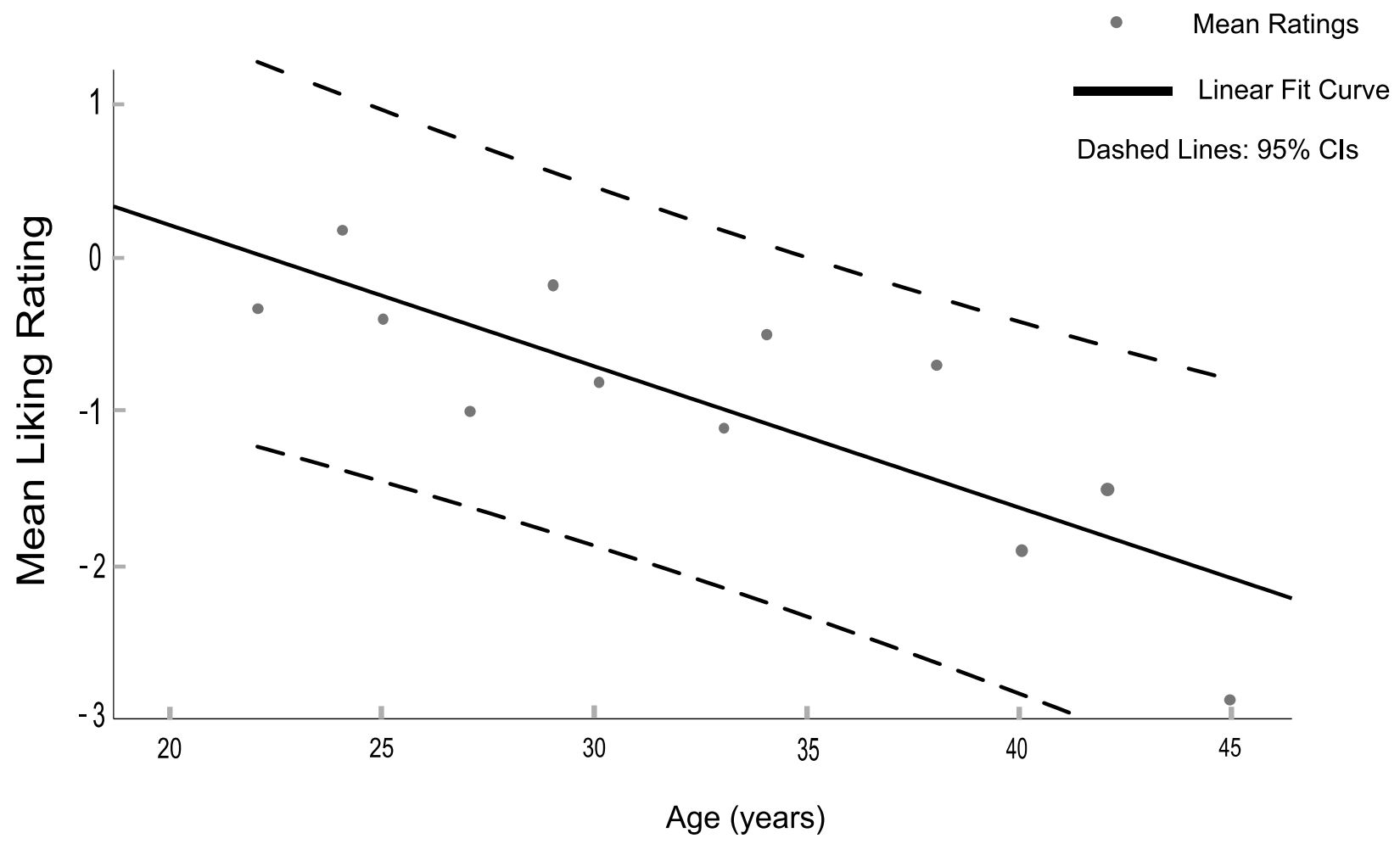

Fig. 6. The relationship between age and liking of food, relative to the background noise in the listening room (Pearson correlation, $r=-0.63, p<0.001$ ). Points represent the mean of all ratings at the age on the $\mathrm{x}$-axis.

(a)

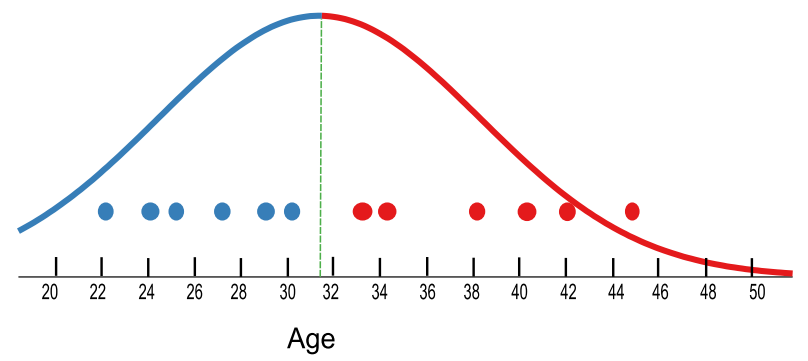

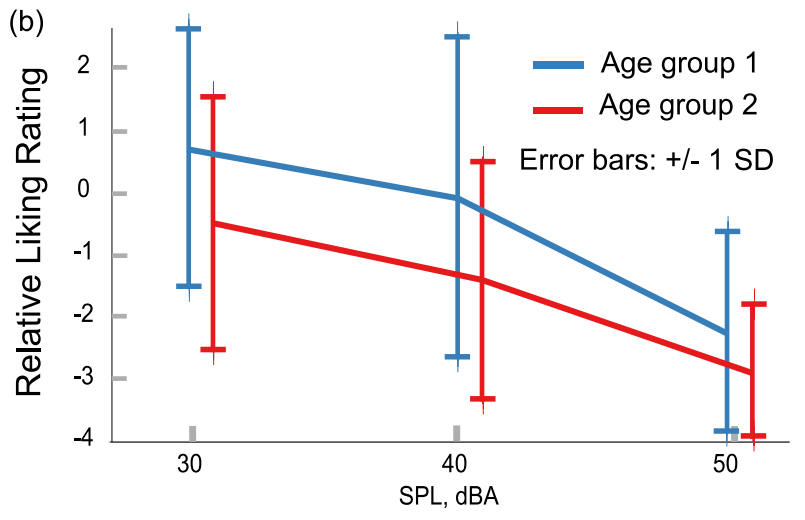

Fig. 7. (a) Age normal distribution of participants showing how they were classified as group 1 and 2. (b) Relationship between the sound pressure level (SPL) and the relative liking of food for groups 1 and 2.

\subsection{The effect of type and level on the relative food liking ratings}

Increasing noise levels decreased the relative liking of food ratings $(F(2,79)=35.4, p<0.001)$. The relative liking ratings of food were higher at $30 \mathrm{dBA}$, compared to 40 and $50 \mathrm{dBA}(p<0.001)$. Noise types had statistically significant effects on the relative liking of food ratings $(F(2,99)=$ 
137.5, $p<0.001)$. Relaxing music stimuli had higher food liking ratings, compared to other types of noise $(p<0.05)$.

\subsection{Interaction effects}

No age vs. gender interaction was observed ( $p>0.05)$; indicating that the gender effect previously described was similar for all age groups. No significant interaction effects were reported between age vs. type, age vs. level, type vs. gender or level vs. gender $(p>0.05)$. However, significant interaction effects were found between the type and level of the masking noise $(F(4,114)=2.65$, $p=0.037$ ); more specifically restaurant stimuli had higher food liking ratings than road traffic noise stimuli at 30 and $40 \mathrm{dBA}(p<0.05)$, while there were no differences between the two noise types at $50 \mathrm{dBA}(p>0.05)$.

\section{Discussion}

Besides the acoustic characteristics of the masking background noise, non-acoustic factors such as gender, age and noise sensitivity can also affect pleasantness to noise (Alamir et al., 2019). Previous studies have only focused on food perception due to the type and level of the noise in the presence of the masking background noise. However, research has not presented the effects of non-acoustic factors such as age and gender on the perception of food in the presence of the background noise. The present study was designed to examine the effect of three non-acoustic factors (i.e. age, gender and noise sensitivity) in the presence of masking background noise on the liking of food. The results suggest that these factors can affect the liking of food significantly.

Annoyance is a subjective measure of the population's reaction to a specific source and it represents the degree of acceptability of that source, rather than its sound energy (Fredianelli et al., 2019). Emotional responses were also found to be correlated with perceived food liking ratings 
(Fiegel et al., 2014). As reported by Kantono et al. (2019), subjectively-rated emotions were correlated with cardiac and skin conductance measures, which were also correlated with the perception of gelato. Therefore, annoyance induced from noise could also affect perceived food liking ratings. In the following discussion of the effect of different factors (i.e. gender, noise sensitivity and age), we will try to show how noise-induced annoyance could mediate the food liking rating due to these factors. The factors associated with food liking are also discussed.

\subsection{The effect of gender on relative food liking}

Gender was found to affect the liking of food ratings. Females gave lower food liking ratings than males, which could be explained by how much each gender is annoyed by the background noise. Females generally have lower hearing thresholds than males in the same age group (Bies et al., 2017; ISO 226:2003 - Acoustics -- Normal equal-loudness-level contours). This acute hearing contributes to a higher perceived annoyance due to the background noise at the same noise level. For example, Radun et al. (2019) investigated the most influential factors to the perception of wind farm noise. Studied responses included subjectively perceived annoyance and sleep disturbance (these responses were acquired using 11-point Likert scales). Gender was the second-highest contributor to annoyance after health concerns and females were more annoyed than males. Therefore, this higher perceived annoyance due to the background noise could contribute to decreased food liking ratings.

One other possible explanation could be that females have a higher health consciousness towards food and noise. Previous studies showed that females have significantly more food and nutritional knowledge, stronger health and dietary beliefs and more physical appearance concerns than males (Turrell, 1997). Females are also generally more health concerned than males (Bower et al., 2003). They also tend to diet more than males and to control food intake for maintaining a slim body 
(Kubberød et al., 2002). Given these concerns, females may also consider the adverse effects of background noise on the liking of food more than males.

The results of food liking due to gender match those observed in earlier studies without including masking background noise. For example, Cordelle et al. (2004) found similar results where women had a slightly lower hedonic taste score or liking score than males.

\subsection{The effect of noise sensitivity on relative food liking}

Noise sensitivity was negatively correlated with relative food liking. A possible explanation for this might be that noise sensitivity is an important factor when studying annoyance elicited by noise in general (Basner et al., 2011; Gille et al., 2017, 2016b). Kantono et al. (2019) showed that these negative emotional responses are correlated with the perception of food. Therefore, this induced annoyance due to increased noise sensitivity could contribute to lower food liking ratings.

\subsection{The effect of age on relative food liking}

The older group of participants had lower ratings of food liking. These differences could be explained in part by the effect of distraction by two different stimuli. The older group may be only focussing on one activity at once. They could be more focused on noise rather than the enjoyment of food. Higher distraction levels are observed for older people compared to younger participants (Leiva et al., 2016). The older group of participants were found to have more time to ignore distracting materials during a reading activity, compared to the younger group (Connelly et al., 1991). It was found recently that older participants were more distracted by acoustic and visual stimuli in a driving activity than middle age groups (Karthaus et al., 2019). The ability to filter out irrelevant information improves from childhood to young adulthood and declines in older age (Wetzel et al., 2006). These results may be also partially explained by the fact that older people 
generally have a decreased taste ability (Kremer et al., 2007; Mojet et al., 2001; Rolls, 1999; Ship, 1999).

\subsection{The effect of level and type of noise on relative food liking}

Listening tests provide controlled stimuli characteristics and confounding factors (Alamir et al., 2018). Characteristics such as noise level and type can be reproduced in a controlled environment such as a listening room (Alamir et al., 2019). This finding supports previous research into food perception which links increased noise level to lower food liking (Spence, 2014; Spence et al., 2019). This may be explained by the fact that background noise can impair our enjoyment of food by masking their auditory impressions (Spence, 2012). Noise type affected the relative liking of the food. This finding is in agreement with Xu's (Xu et al., 2019) findings which showed that positive emotions were reported in the presence of café noise mixed with forest and bird soundscapes as opposed to when mixed with machine noise.

\subsection{Future studies and limitations}

The generalizability of the current results is subject to certain limitations. For instance, with small sample size, caution must be applied, as the findings might not be transferable to different categories of participants (e.g. normal hearing participants only were included). Therefore, more experiments with a large sample size including different participants' groups could establish these effects.

This study presented the overall subjective experience of food in the presence of masking background noise (i.e. liking of food). Background noise could also affect other specific sensory outcomes of food (Lin et al., 2019). The overall hedonic valence and arousal impression of noise stimuli could have made it clearer to interpret the current results. However, the question about the 
arousal impression of the masking background noise might have emphasized the possible role of the sound on food evaluation by directing participants' attention toward the background noise. The variant responses in measurements of pleasantness or the liking of the food in crossmodal influences (e.g. chemo-senses and the auditory sense) are also important as one sense will be more dominant (Kantono et al., 2016b, 2016c). In this case, the auditory system will be more dominant when rating temporal events (Spence and Shankar, 2010). Therefore, it would be also useful to consider the elicited perception of different stimuli and their temporal variations (Fiegel et al., 2014).

The highest levels of noise presented in this study ( $50 \mathrm{dBA})$ could be lower than the realistic levels found in some countries such as USA (Rusnock and Bush, 2012) and China (To and Chung, 2014). However, it should be noted that $40 \mathrm{dBA}$ is the recommended limit for restaurants (Bies et al., 2017).

Response differences depend on the eating contexts. Kantono et al. (2018) showed that perceived cocoaness, sweetness, and milkiness of gelato were reported more in the natural eating environment than the laboratory setting, while bitterness and creaminess were the least reported in these contexts. Similar sensory attributes were found in immersive and natural eating environments. It would be useful to investigate multiple interactions such as external visual effects of the dining areas besides different acoustic characteristics of the stimuli. For example, Kantono et al. (2016a) found that pleasantness ratings of chocolate gelati increased in the presence of audiovisual cues compared to auditory cues only. Therefore, it would also be useful to determine the relative importance of different external visual cues for food enjoyment.

Apart from acoustic characteristics which have been shown to affect the response considerably, this paper complements previous studies by showing the potential of non-acoustic factors to affect 
responses. The study did not evaluate other confounding factors such as hearing acuity. In future investigations, further non-acoustic factors could be investigated through laboratory studies. Therefore, the models of food perception and its contributing factors can be determined.

Other acoustic factors could affect the perception of food. For example, signal to noise ratio is an acoustic term that has psychophysical effects, and it represents the degree by which the masking background noise is dominant over the ambient background noise (Fastl and Zwicker, 2001). The same level of the overall background noise can be achieved at different dominance ratios of masking noise (e.g. music), compared to the original background noise in the room. Further research should be done to investigate how this ratio can be a potential factor in food perception.

\subsection{Implications}

The findings of this study help us to understand how background noise affects food perception by considering both acoustic and non-acoustic factors. This can help develop food perception models in the presence of background noise. This can also provide scientific fact-based evidence of food perception in the presence of background noise. For example, the background noise of the dining areas for younger age people could be different from other people as age is a significant factor affecting food perception and older participants could have worse dining experience in the presence of high background noise. Another implication of this is the possibility that different places in dining areas could be also provided based on the preference of people or how they are sensitised to noise.

A better understanding of these factors will likely lead to future practical applications. For example, poor service has been found to be the most annoying complaint in restaurants (Spence, 2014). This study helps provide education for food providers. If they know that psychological 
factors such as age, noise sensitivity and gender can modulate changes in food liking, better service can be provided in terms of dealing with people and better noise management in dining areas. 


\section{Conclusion}

This study showed that age, gender and noise sensitivity affected the food liking significantly in the presence of background noise. Gender had a significant effect on food liking. In particular, the food liking ratings of males were higher than the ratings of females. The increase in noise sensitivity and age led to lower food liking ratings. The sensitive group gave lower ratings of food liking than the insensitive group. The older group of participants gave lower ratings of food liking.

Mechanisms that could mediate the perceived food liking in the presence of background noise due to the three factors studied (i.e. age, gender and noise sensitivity) were discussed. A possible mechanism could be the perceived annoyance from the background noise. In previous studies, negative emotions were consistently correlated with a decreased perceived liking of food. That could explain why noise-sensitive participants had lower food liking ratings. Moreover, distraction due to age could make older participants focus only on one activity. This could also reduce the enjoyment of food for older participants besides their low taste ability in the presence of background noise. Another proposed possible mechanism was related to gender differences. For example, females have different expectations than males in terms of more food and nutritional knowledge, stronger health and dietary beliefs and physical appearance concerns. Females also have more acute hearing than males in the same age group, which can lead to increased annoyance at the same noise level. This led to reduced relative food liking ratings for females, compared to males.

The findings of this study have important implications for providing a better dining experience through noise management. For example, quiet dining areas should be considered for old and noise-sensitive people. This also provides practical insights into understanding how non-acoustic factors such as gender can affect food perception to provide adequate education for food providers. 


\section{References}

Alamir, M.A., Hansen, K.L., Zajamsek, B., 2018. The effect of wind farm noise on human response: An analysis of listening test methodologies, in: Proceedings of ACOUSTICS 2018. Adelaide, Australia, pp. 1-9.

Alamir, M.A., Hansen, K.L., Zajamsek, B., Catcheside, P., 2019. Subjective responses to wind farm noise: A review of laboratory listening test methods. Renew. Sustain. Energy Rev. 114, 109317. https://doi.org/10.1016/j.rser.2019.109317

Basner, M., Müller, U., Elmenhorst, E.-M., 2011. Single and combined effects of air, road, and rail traffic noise on sleep and recuperation. Sleep 34, 11-23. https://doi.org/Research Support, Non-U.S. Gov’t

Bies, D.A., Hansen, C.H., Howard, C., 2017. Engineering Noise Control, CRC Press. https://doi.org/10.3397/1.3455051

Bower, J.A., Saadat, M.A., Whitten, C., 2003. Effect of liking, information and consumer characteristics on purchase intention and willingness to pay more for a fat spread with a proven health benefit. Food Qual. Prefer. 14, 65-74. https://doi.org/10.1016/S09503293(02)00019-8

Connelly, S.L., Hasher, L., Zacks, R.T., 1991. Age and reading: the impact of distraction. Psychol. Aging 6, 533-541. https://doi.org/10.1037/0882-7974.6.4.533

Crisinel, A.S., Spence, C., 2010. A sweet sound? Food names reveal implicit associations between taste and pitch. Perception 39, 417-425. https://doi.org/10.1068/p6574

DeCoster, J., Iselin, A.M.R., Gallucci, M., 2009. A Conceptual and Empirical Examination of Justifications for Dichotomization. Psychol. Methods 14, 349-366. https://doi.org/10.1037/a0016956

Duizer, L., 2001. A review of acoustic research for studying the sensory perception of crisp, crunchy and crackly textures. Trends Food Sci. Technol. 12, 17-24. https://doi.org/10.1016/S0924-2244(01)00050-4

Fastl, H., Zwicker, E., 2001. Psychoacoustics: Facts and Models. https://doi.org/10.1007/978-3642-88163-3

Fiegel, A., Meullenet, J.F., Harrington, R.J., Humble, R., Seo, H.S., 2014. Background music genre can modulate flavor pleasantness and overall impression of food stimuli. Appetite 76, 144-152. https://doi.org/10.1016/j.appet.2014.01.079

Fredianelli, L., Carpita, S., Licitra, G., 2019. A procedure for deriving wind turbine noise limits by taking into account annoyance. Sci. Total Environ. 648, 728-736.

https://doi.org/10.1016/j.scitotenv.2018.08.107

Gille, L.A., Marquis-Favre, C., Morel, J., 2016a. Testing of the European Union exposureresponse relationships and annoyance equivalents model for annoyance due to transportation noises: The need of revised exposure-response relationships and annoyance equivalents model. Environ. Int. 94, 83-94. https://doi.org/10.1016/j.envint.2016.04.027 
Gille, L.A., Marquis-Favre, C., Weber, R., 2017. Aircraft noise annoyance modeling: Consideration of noise sensitivity and of different annoying acoustical characteristics. Appl. Acoust. 115, 139-149. https://doi.org/10.1016/j.apacoust.2016.08.022

Gille, L.A., Marquis-Favre, C., Weber, R., 2016b. Noise sensitivity and loudness derivative index for urban road traffic noise annoyance computation. J. Acoust. Soc. Am. 140, $4307-$ 4317. https://doi.org/10.1121/1.4971329

Iacobucci, D., Posavac, S.S., Kardes, F.R., Schneider, M.J., Popovich, D.L., 2015. Toward a more nuanced understanding of the statistical properties of a median split. J. Consum. Psychol. 25, 652-665. https://doi.org/10.1016/j.jcps.2014.12.002

ISO 226:2003 - Acoustics -- Normal equal-loudness-level contours, n.d.

Kantono, K., Hamid, N., Shepherd, D., Lin, Y.H.T., Brard, C., Grazioli, G., Thomas Carr, B., 2018. The effect of music on gelato perception in different eating contexts. Food Res. Int. 113, 43-56. https://doi.org/10.1016/j.foodres.2018.06.030

Kantono, K., Hamid, N., Shepherd, D., Lin, Y.H.T., Skiredj, S., Carr, B.T., 2019. Emotional and electrophysiological measures correlate to flavour perception in the presence of music. Physiol. Behav. 199, 154-164. https://doi.org/10.1016/j.physbeh.2018.11.012

Kantono, K., Hamid, N., Shepherd, D., Lin, Y.H.T., Yakuncheva, S., Yoo, M.J.Y., Grazioli, G., Carr, B.T., 2016a. The influence of auditory and visual stimuli on the pleasantness of chocolate gelati. Food Qual. Prefer. 53, 9-18. https://doi.org/10.1016/j.foodqual.2016.05.008

Kantono, K., Hamid, N., Shepherd, D., Yoo, M.J.Y., Carr, B.T., Grazioli, G., 2016b. The effect of background music on food pleasantness ratings. Psychol. Music 44, 1111-1125. https://doi.org/10.1177/0305735615613149

Kantono, K., Hamid, N., Shepherd, D., Yoo, M.J.Y., Grazioli, G., Carr, B.T., 2016c. Listening to music can influence hedonic and sensory perceptions of gelati. Appetite 100, 244-255. https://doi.org/10.1016/j.appet.2016.02.143

Karthaus, M., Wascher, E., Falkenstein, M., Getzmann, S., 2019. The ability of young, middleaged and older drivers to inhibit visual and auditory distraction in a driving simulator task. Transp. Res. Part F Traffic Psychol. Behav. 68, 272-284. https://doi.org/10.1016/j.trf.2019.11.007

Kremer, S., Mojet, J., Kroeze, J.H.A., 2007. Differences in perception of sweet and savoury waffles between elderly and young subjects. Food Qual. Prefer. 18, 106-116. https://doi.org/10.1016/j.foodqual.2005.08.007

Kubberød, E., Ueland, Ø., Tronstad, Å., Risvik, E., 2002. Attitudes towards meat and meateating among adolescents in Norway: A qualitative study. Appetite 38, 53-62. https://doi.org/10.1006/appe.2002.0458

Leiva, A., Andrés, P., Servera, M., Verbruggen, F., Parmentier, F.B.R., 2016. The role of age, working memory, and response inhibition in deviance distraction: A cross-sectional study. Dev. Psychol. 52, 1381-1393. https://doi.org/10.1037/dev0000163 
Lin, Y.H.T., Hamid, N., Shepherd, D., Kantono, K., Spence, C., 2019. Environmental sounds influence the multisensory perception of chocolate gelati. Foods 8, 1-19. https://doi.org/10.3390/foods8040124

Littell, R.C., Pendergast, J., Natarajan, R., 2000. Mixed Models: Modelling Covariance Structure in the Analysis of Repeated Measures Data. Stat. Med. 19, 1793-1819.

https://doi.org/10.1002/0470023724.ch1c(ii)

Macht, M., 2008. How emotions affect eating: A five-way model. Appetite 50, 1-11. https://doi.org/10.1016/j.appet.2007.07.002

Mathias Basner, Wolfgang Babisch, Adrian Davis, Mark Brink, Sabine Janssen, Stephen Stansfeld, S.S., 2014. Auditory and non-auditory effects of noise on health Mathias. Lancet. Author Manuscr. 12, 1325-1332. https://doi.org/10.1016/S0140-6736(13)61613-X.Auditory

Mojet, J., Christ-Hazelhof, E., Heidema, J., 2001. Taste Perception with Age: Generic or Specific Losses in Threshold Sensitivity to the Five Basic Tastes? Chem. Senses 26, 845860. https://doi.org/10.1093/chemse/26.7.845

Münzel, T., Schmidt, F.P., Steven, S., Herzog, J., Daiber, A., Sørensen, M., 2018 a. Environmental Noise and the Cardiovascular System. J. Am. Coll. Cardiol. 71, 688-697. https://doi.org/10.1016/j.jacc.2017.12.015

Münzel, T., Sørensen, M., Schmidt, F., Schmidt, E., Steven, S., Kröller-Schön, S., Daiber, A., 2018b. The Adverse Effects of Environmental Noise Exposure on Oxidative Stress and Cardiovascular Risk. Antioxid. Redox Signal. 28, 873-908. https://doi.org/10.1089/ars.2017.7118

Pawlaczyk-Łuszczyńska, M., Zaborowski, K., Dudarewicz, A., Zamojska-Daniszewska, M., Waszkowska, M., 2018. Response to Noise Emitted by Wind Farms in People Living in Nearby Areas. Int. J. Environ. Res. Public Health 15, 1575. https://doi.org/10.3390/ijerph15081575

Poulsen, A.H., Raaschou-Nielsen, O., Peña, A., Hahmann, A.N., Nordsborg, R.B., Ketzel, M., Brandt, J., Sørensen, M., 2018. Long-term exposure to wind turbine noise at night and risk for diabetes: A nationwide cohort study. Environ. Res. 165, 40-45. https://doi.org/10.1016/j.envres.2018.03.040

Radun, J., Hongisto, V., Suokas, M., 2019. Variables associated with wind turbine noise annoyance and sleep disturbance. Build. Environ. 150, 339-348. https://doi.org/10.1016/j.buildenv.2018.12.039

Rolls, B.J., 1999. Do chemosensory changes influence food intake in the elderly? Physiol. Behav. 66, 193-197. https://doi.org/10.1016/S0031-9384(98)00264-9

Rusnock, C.F., Bush, P.M.C., 2012. An evaluation of restaurant noise levels and contributing dactors. J. Occup. Environ. Hyg. 9. https://doi.org/10.1080/15459624.2012.683716

Schäffer, B., Schlittmeier, S.J., Pieren, R., Heutschi, K., Brink, M., Graf, R., Hellbrück, J., 2016. Short-term annoyance reactions to stationary and time-varying wind turbine and road traffic noise: A laboratory study. J. Acoust. Soc. Am. 139, 2949-2963. https://doi.org/10.1121/1.4949566 
Ship, J.A., 1999. The influence of aging on oral health and consequences for taste and smell. Physiol. Behav. 66, 209-215. https://doi.org/10.1016/S0031-9384(98)00267-4

Spence, C., 2014. Noise and its impact on the perception of food and drink. Flavour 3, 1-17. https://doi.org/10.1186/2044-7248-3-9

Spence, C., Reinoso-Carvalho, F., Velasco, C., Wang, Q.J., 2019. Extrinsic auditory contributions to food perception \& Consumer behaviour: An interdisciplinary review. Multisens. Res. 20, 1-44. https://doi.org/10.1163/22134808-20191403

Spence, C., Shankar, M.U., 2010. The influence of auditory cues on the perception of, and responses to, food and drink. J. Sens. Stud. 25, 406-430. https://doi.org/10.1111/j.1745459X.2009.00267.x

To, W.M., Chung, A., 2014. Noise in restaurants: Levels and mathematical model. Noise Heal. 16.

Turrell, G., 1997. Determinants of gender differences in dietary behavior. Nutr. Res. 17, $1105-$ 1120. https://doi.org/10.1021/ac00253a600

Weinstein, N.D., 1978. Individual differences in reactions to noise: A longitudinal study in a college dormitory. J. Appl. Psychol. 63, 458-466.

Wesson, D.W., Wilson, D.A., 2010. Smelling sounds: Olfactory-auditory sensory convergence in the olfactory tubercle. J. Neurosci. 30, 3013-3021. https://doi.org/10.1523/JNEUROSCI.6003-09.2010

Wetzel, N., Widmann, A., Berti, S., Schröger, E., 2006. The development of involuntary and voluntary attention from childhood to adulthood: A combined behavioral and event-related potential study. Clin. Neurophysiol. 117, 2191-2203.

https://doi.org/10.1016/j.clinph.2006.06.717

Woods, A.T., Poliakoff, E., Lloyd, D.M., Kuenzel, J., Hodson, R., Gonda, H., Batchelor, J., Dijksterhuis, G.B., Thomas, A., 2011. Effect of background noise on food perception. Food Qual. Prefer. 22, 42-47. https://doi.org/10.1016/j.foodqual.2010.07.003

Xu, Y., Hamid, N., Shepherd, D., Kantono, K., Spence, C., 2019. Changes in flavour, emotion, and electrophysiological measurements when consuming chocolate ice cream in different eating environments. Food Qual. Prefer. 77, 191-205. https://doi.org/10.1016/j.foodqual.2019.05.002 